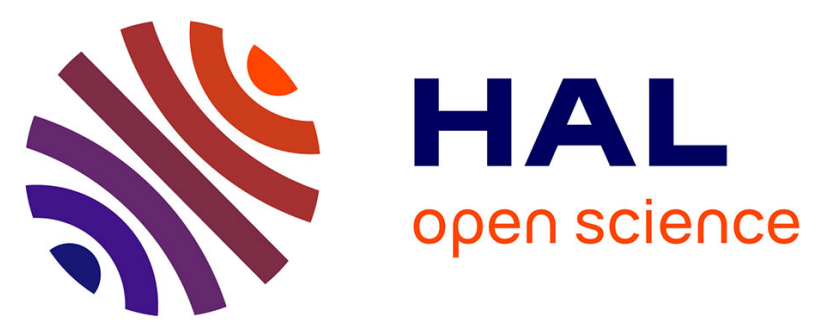

\title{
Direct observation of field and temperature induced domain replication in dipolar coupled perpendicular anisotropy films
}

Thomas Hauet, C.M. Günther, B Pfau, M.E. Schabes, J.-U Thiele, Rick R.L., P Fischer, S Eisebitt, O Hellwig

\section{To cite this version:}

Thomas Hauet, C.M. Günther, B Pfau, M.E. Schabes, J.-U Thiele, et al.. Direct observation of field and temperature induced domain replication in dipolar coupled perpendicular anisotropy films. Physical Review B: Condensed Matter and Materials Physics (1998-2015), 2008, 10.1103/PhysRevB.77.184421 . hal-01344807

\section{HAL Id: hal-01344807 https://hal.science/hal-01344807}

Submitted on 12 Jul 2016

HAL is a multi-disciplinary open access archive for the deposit and dissemination of scientific research documents, whether they are published or not. The documents may come from teaching and research institutions in France or abroad, or from public or private research centers.
L'archive ouverte pluridisciplinaire HAL, est destinée au dépôt et à la diffusion de documents scientifiques de niveau recherche, publiés ou non, émanant des établissements d'enseignement et de recherche français ou étrangers, des laboratoires publics ou privés. 


\title{
Direct observation of field and temperature induced domain replication in dipolar coupled perpendicular anisotropy films
}

\author{
T. Hauet, ${ }^{1}$ C. M. Günther, ${ }^{2}$ B. Pfau, ${ }^{2}$ M. E. Schabes, ${ }^{1}$ J.-U. Thiele, ${ }^{1}$ R. L. Rick,,${ }^{3,4}$ P. Fischer, ${ }^{5}$ S. Eisebitt, ${ }^{2}$ and O. Hellwig ${ }^{1}$ \\ ${ }^{1}$ San Jose Research Center, Hitachi Global Storage Technologies, San Jose, California 95135, USA \\ ${ }^{2}$ BESSY mbH, 12489 Berlin, Germany \\ ${ }^{3}$ Department of Applied Physics, Stanford University, Stanford, California 94305, USA \\ ${ }^{4}$ Stanford Synchrotron Radiation Laboratory, SLAC, Menlo Park, California 94025, USA \\ ${ }^{5}$ Center for X-Ray Optics, Lawrence Berkeley National Laboratory, Berkeley, California 94720 USA
}

(Received 2 January 2008; published 19 May 2008)

\begin{abstract}
Dipolar interactions in a soft/Pd/hard $[\mathrm{CoNi} / \mathrm{Pd}]_{30} / \mathrm{Pd} /[\mathrm{Co} / \mathrm{Pd}]_{20}$ multilayer system, where a thick Pd layer between two ferromagnetic units prevents direct exchange coupling, are directly revealed by combining magnetometry and state-of-the-art layer resolving soft x-ray imaging techniques with sub-100-nm spatial resolution. The domains forming in the soft layer during external magnetic field reversal are found to match the domains previously trapped in the hard layer. The low Curie temperature of the soft layer allows varying its intrinsic parameters via temperature and thus studying the competition with dipolar fields due to the domains in the hard layer. Micromagnetic simulations elucidate the role of [CoNi/Pd] magnetization, exchange, and anisotropy in the duplication process. Finally, thermally driven domain replication in remanence during temperature cycling is demonstrated.
\end{abstract}

DOI: 10.1103/PhysRevB.77.184421

PACS number(s): 75.70.Kw, 75.60.Jk, 75.70.Cn

Dipolar interactions in layered magnetic systems have recently attracted increasing attention in order to improve the reliability of magnetoelectronic devices ${ }^{1}$ and for applications in multilevel magnetic recording. ${ }^{2}$ In this context, stray field induced replication of domains or bit patterns in perpendicular anisotropy systems has been investigated as well. ${ }^{3}$ In previous studies, standard techniques such as magnetometry, magnetic force microscopy (MFM) or Kerr microscopy have been used to characterize the evolution of the magnetic configuration during field cycling at room temperature. ${ }^{3,4}$ However, such techniques average over the complete ferromagnetic layer stack and do not allow studying the hard and soft layer separately. Thermally induced magnetization reversal with or without an external static magnetic field is nowadays under heavy discussion since it is being considered for applications in magnetic random access memory or magnetooptical (MO) recording devices. ${ }^{5}$ Domain replication has already been proposed as a readout mechanism for $\mathrm{MO}$ recording in magnetic amplifying magneto-optical systems, but it is also believed to be useful for thermally induced writing. 6

In the present paper, we report on high-resolution imaging of the domain replication in an all-perpendicular anisotropy system. Using a soft/Pd/hard multilayer system, namely, $[\mathrm{CoNi} / \mathrm{Pd}]_{30} / \mathrm{Pd} /[\mathrm{Co} / \mathrm{Pd}]_{20}$, we combine magnetometry and state-of-the-art element-specific $\mathrm{X}$-ray imaging techniques ${ }^{7-9}$ to investigate the magnetic configuration of the hard and soft layers independently during external magnetic field cycles at temperatures in the range of 150 up to $350 \mathrm{~K}$. Field cycles lead to the formation of a soft layer magnetic domain state that is identical to the domain configuration previously trapped in the hard layer. Furthermore, we exploit the strong temperature dependence of the $[\mathrm{CoNi} / \mathrm{Pd}]$ soft layer to study the domain duplication as a function of temperature, and demonstrate thermally driven domain replication at remanence.
Using dc magnetron sputtering, the layer sequence $\operatorname{Pd}(3 \mathrm{~nm}) /\left[\mathrm{Co}_{30} \mathrm{Ni}_{70}(0.2 \mathrm{~nm}) / \mathrm{Pd}(1.5 \mathrm{~nm})\right]_{30} /$ $\operatorname{Pd}(10 \mathrm{~nm}) /[\mathrm{Co}(0.3 \mathrm{~nm}) / \operatorname{Pd}(0.8 \mathrm{~nm})]_{20} / \operatorname{Pd}(1.2 \mathrm{~nm})$ is deposited onto $\mathrm{Si}$ wafers as well as $\mathrm{Si}_{3} \mathrm{~N}_{4}$ membranes for transmission soft $\mathrm{x}$-ray imaging. The macroscopic magnetic properties are measured using vibrating sample magnetometry (VSM). Figure 1(a) compares hard and soft multilayer magnetizations as a function of temperature. The hard $[\mathrm{Co} / \mathrm{Pd}]$ system, deposited at $7 \mathrm{mTorr}$ Ar pressure, reveals a welldefined perpendicular-to-the-sample-plane anisotropy and an almost constant magnetization within the considered temperature range from 5 to $400 \mathrm{~K}$. However, the Ni concentration in the soft $[\mathrm{CoNi} / \mathrm{Pd}]$, deposited at 3 mTorr Ar pressure, is tuned to obtain a Curie temperature $\left(T_{C}\right)$ close to $350 \mathrm{~K}$, i.e., its magnetization and anisotropy [Fig. 1(b)] decrease continuously from 5 to $350 \mathrm{~K}$, and the system turns paramagnetic for higher temperatures. ${ }^{10}$ We measure the temperature

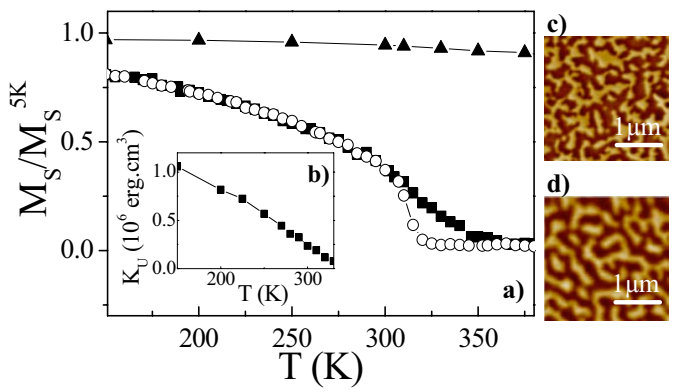

FIG. 1. (Color online) (a) Normalized magnetization versus temperature profiles of a $\left[\mathrm{Co}_{30} \mathrm{Ni}_{70}(0.2 \mathrm{~nm}) / \mathrm{Pd}(1.5 \mathrm{~nm})\right]_{30}$ soft multilayer measured under 0 Oe (open circles) and $1 \mathrm{kOe}$ (solid squares) field applied perpendicular to the film plane and of a $[\mathrm{Co}(0.3 \mathrm{~nm}) / \operatorname{Pd}(0.8 \mathrm{~nm})]_{20}$ hard multilayer at remanence (triangles). (b) Temperature dependence of the anisotropy for the [CoNi/Pd] soft layer. (c),(d) $2.5 \times 2.5 \mu^{2}$ MFM images of demagnetized $[\mathrm{Co} / \mathrm{Pd}]$ and $[\mathrm{CoNi} / \mathrm{Pd}]$ multilayers, respectively. 

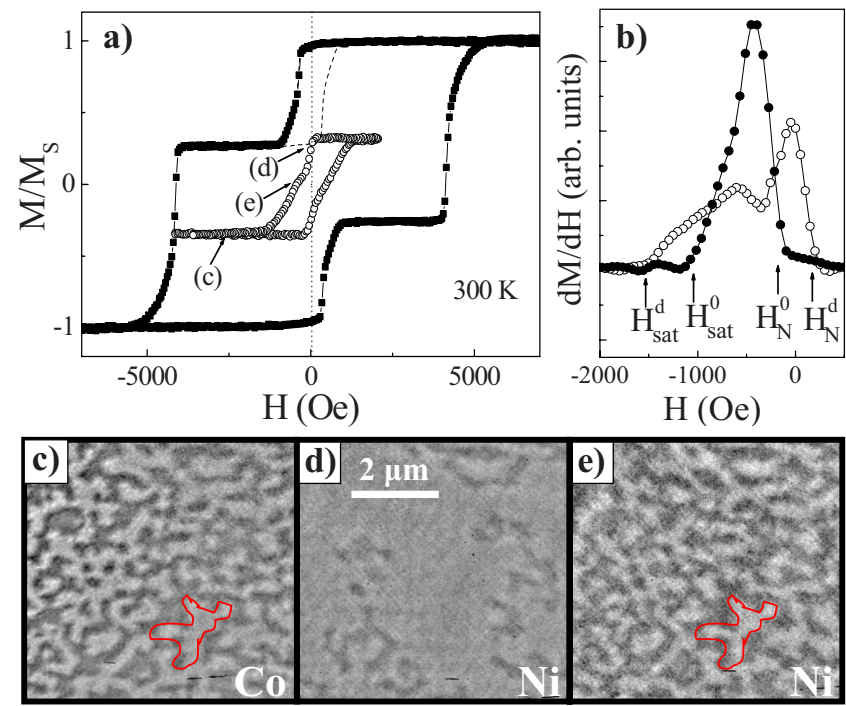

FIG. 2. (Color online) (a) Normalized hysteresis loops measured at $300 \mathrm{~K}$. Square solid symbols show the major loop, while the dashed line and open circles correspond to minor loops with the hard layer in a uniform and a domain state, respectively. (b) Derivative of the descending major and minor (solid and open symbols) hysteresis loop branches. (c)-(e) MTXM images, each $6 \times 6 \mu \mathrm{m}^{2}$ showing the magnetic domain configuration as observed for the three states marked in (a), respectively. Image (c) is collected at the Co $L_{3}$ edge $(778 \mathrm{eV})$ while images (d) and (e) are obtained at the $\mathrm{Ni} L_{3}$ edge $(854 \mathrm{eV})$. Red lines are a guide to the eye.

dependence of the magnetization at remanence as well as in an external field of $1 \mathrm{kOe}$ and find slight differences between 310 and $350 \mathrm{~K}$, indicating that the out-of-plane anisotropy competes with an in-plane shape anisotropy when approaching $T_{C}$ [Fig. 1(a)].

Figure 2(a) presents hysteresis loops measured at $300 \mathrm{~K}$ with the field perpendicular to the sample plane. Starting from positive saturation, the major loop reveals two distinct steps corresponding to the independent reversal of the soft (at $H_{N}^{0}=-350 \mathrm{Oe}$ ) and the hard (at $H=-4 \mathrm{kOe}$ ) layers. A minor field cycle performed between $+/-2 \mathrm{kOe}$ shows no residual bias field and indicates that the $\mathrm{Pd}(10 \mathrm{~nm})$ layer in between the two ferromagnetic multilayers prevents any direct exchange interaction. Sweeping the external perpendicular magnetic field from 8 to $-4.2 \mathrm{kOe}$, where the hard layer has reversed half of its magnetization, we create a domain state in the hard layer with about $50 \%$ up and $50 \%$ down domains. To directly image the hard and soft layer domains with sub-100-nm spatial resolution, we performed magnetic transmission soft x-ray microscopy (MTXM) using the full field soft X-ray setup of beamline 6.1.2. at the Advanced Light Source (ALS) in Berkeley, CA. ${ }^{11,12}$ X-ray magnetic circular dichroism (XMCD) provides element-specific magnetic contrast, which allows separate imaging of the hard and soft layer magnetic domain configuration by tuning the photon energy to the $\mathrm{Co}$ and $\mathrm{Ni} L_{3}$ absorption edges, respectively. MTXM images were recorded with magnetic fields up to $2 \mathrm{kOe}$ pointing perpendicular to the sample plane. After reversing half of the hard layer magnetization, we confirm [Fig. 2(c)] the presence of a $50 \%$ up- $50 \%$ down labyrinth domain pattern similar to that previously obtained via MFM [Figs. 1(c) and 1(d)]. Since the soft layer magnetization is saturated for Fig. 2(c), the domain configuration measured at the Co $L_{3}$ edge at -2 kOe reflects the hard layer state only.

Subsequently we measure the reversal of the soft layer in a minor loop between $+/-2$ kOe [open circles in Fig. 2(a)]. Corresponding MTXM images were obtained at the $\mathrm{Ni} L_{3}$ edge, to separate out the reversal of the soft layer only [Figs. 2(d) and 2(e)]. Starting from positive soft layer saturation, we observe domain nucleation in the soft layer [Fig. 2(d)] around 0 Oe in good agreement with the VSM measurement [Fig. 2(a)]. The nucleation field $\left(H_{N}\right)$ and the saturation field $\left(H_{S}\right)$ of the soft layer have significantly changed as compared to the previous minor loop measured for a uniform hard layer. This is more clearly visible in the derivatives of the descending hysteresis branches in Fig. 2(b). $H_{N}$ increases from $H_{N}^{0}=-250$ Oe to $H_{N}^{d}=+130 \mathrm{Oe}$, while $H_{S}$ decreases from $H_{S}^{0}=-1.1 \mathrm{kOe}$ to $H_{S}^{d}=-1.55 \mathrm{kOe}$. Such changes in the soft layer hysteresis loop have already been reported on similar hard-soft systems and were assumed to originate from the influence of dipolar fields induced by the hard layer domains on the soft layer reversal. ${ }^{3}$ At about -300 Oe we reach a $50 \%$ up and $50 \%$ down domain state [Fig. 2(e)] that appears to match very well with the state previously trapped in the hard layer [Fig. 2(c)]. At this stage of the minor loop the domain state of the hard layer has completely been copied (replicated) into the soft layer. In Fig. 2(b), this replicated state reveals itself as a dip in the derivative. We find symmetric minor loops indicating close to perfect return point and complementary point memory in the soft layer. ${ }^{13}$ However, while the same replicated domain state was observed during several successive field loops, we found no evidence for an identical nucleation sequence.

In order to go beyond this room temperature (RT) study we used the recently established lensless Fourier transform holography (FTH) technique, ${ }^{8}$ which allows investigating the domain replication process layer resolved (XMCD) at variable temperature. The sample is illuminated with coherent $\mathrm{x}$ rays through a gold mask with a circular $1.2-\mu$ m-diameter object aperture and a $\sim 100 \mathrm{~nm}$ reference hole to create an $\mathrm{x}$-ray hologram that contains the relative phase between object and reference and thus, when transformed back into real space, yields a direct image of the object aperture domain pattern. Corresponding experiments were performed at beamline UE-52-SGM at BESSY in Berlin (Germany) using the ALICE setup, ${ }^{14}$ which allows holographic imaging at variable sample temperature and under external fields up to 7 kOe. More details about magnetic FTH imaging can be found elsewhere. ${ }^{8,9}$ Using FTH imaging at lower temperature $(150 \mathrm{~K})$ we find similar domain replication behavior from the hard into the soft layer to that previously observed via MTXM at RT. A careful analysis of the derivatives of the descending VSM hysteresis loop branches for various temperature reveals that the amplitudes of $H_{N}$ and $H_{\text {sat }}$ variations due to the domain state in the hard layer differ with temperature. As shown in Fig. 3, the absolute values of $\Delta H_{N}=H_{N}^{d}-H_{N}^{0}$ and $\Delta H_{\text {sat }}=H_{\text {sat }}^{d}-H_{\text {sat }}^{0}$ increase as the temperature is raised. The variation of $\Delta H_{N}$ from 100 to $310 \mathrm{~K}$ is two times larger than that of $\Delta H_{\text {sat }}$ over the same temperature range. 

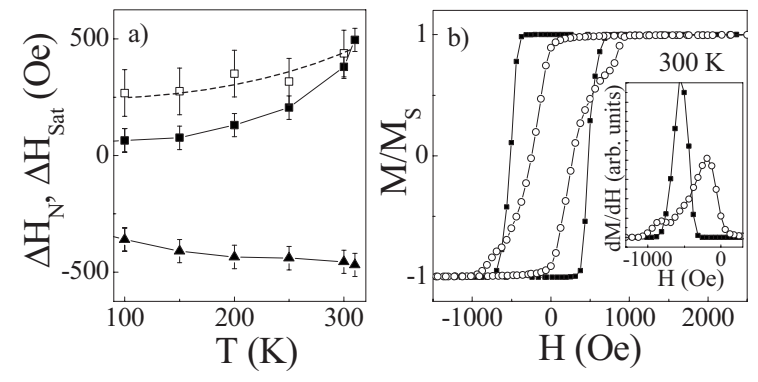

FIG. 3. (a) Experimental nucleation and saturation field difference, $\Delta H_{N}$ (solid square) and $\Delta H_{\text {sat }}$ (triangle), for the soft layer minor loops with and without domains in the hard layer as a function of temperature. Open symbols correspond to the simulated $\Delta H_{N}$ with the dashed line being a guide to the eye. (b) Simulation of $300 \mathrm{~K}$ soft layer hysteresis loops considering a uniform (square) or stripe domain (circle) hard layer state, and their derivatives (inset).

We performed micromagnetic simulations to investigate the origin of the nucleation field reduction in the soft layer due to the domains in the hard layer. The hysteresis loop of the $[\mathrm{CoNi} / \mathrm{Pd}]$ is calculated from the solution of the LandauLifshitz-Gilbert (LLG) equations ${ }^{15}$ with the $[\mathrm{Co} / \mathrm{Pd}]$ magnetization held fixed in either the uniform state or the stripe domain configuration. In the simulations the domains in the hard layer are represented by $200 \times 3000 \mathrm{~nm}^{2}$ parallel stripes of alternating magnetization perpendicular to the plane of the film. The $\mathrm{CoNi} / \mathrm{Pd}$ layer is first uniformly magnetized at $+7.5 \mathrm{kOe}$, then the applied field is swept between $+/-2.5 \mathrm{kOe}$ and superimposed on the magnetostatic interaction fields from the hard layer domains. The sweep rate of the field is sufficiently slow $(6.4 \mathrm{~ns} / \mathrm{kOe})$ at a gyromagnetic damping $\alpha=0.1$ to avoid cases where dynamic effects would dominate the switching behavior. Thermal fluctuations are not considered explicitly in the LLG equations, but are lumped into adjustments of the values for the magnetization $M_{s}$, the uniaxial anisotropy $K_{1}$, and the exchange $A$ in the soft layer as a function of temperature. The modeling of the experimental nucleation fields proceeds in two steps. First, we aim to match the $H_{N}^{0}$ (without stripe domains) by adjusting the $[\mathrm{CoNi} / \mathrm{Pd}]$ parameters to obtain effective values for the anisotropy $K_{\text {eff }}$ and the exchange $A_{\text {eff }}$ as a function of temperature. Second, the calculations are repeated with $K_{\text {eff }}$ and $A_{\text {eff }}$ to obtain the nucleation field $H_{N}^{d}$ when stripe domains are present in the hard layer to finally estimate the reduction in nucleation field, $\Delta H_{N}$. We find that it is generally possible to qualitatively obtain the loop shape of Fig. 2 [see Fig. 3(b)]; however, $K_{\text {eff }}$ has to be significantly lowered compared to the experimental anisotropy measured from the hard axis loops [Fig. 1(b)]. Typically $K_{\text {eff }}$ was $25-30 \%$ of the experimental $K$ with $A_{\text {eff }}$ in the range of $(1.1-1.5) \times 10^{-11} \mathrm{~J} / \mathrm{m}$ at $T=150 \mathrm{~K}$, and $(0.1-0.5) \times 10^{-11} \mathrm{~J} / \mathrm{m}$ at $T=300 \mathrm{~K}$. Such low values of $K_{\text {eff }}$ in the soft layer also require a scaling of the duplication fields by a factor of $\sim 0.35$ (e.g., by a commensurate adjustment of the hard layer magnetization). The lack of additional information at the microstructural level, e.g., pinning-site density in $[\mathrm{CoNi} / \mathrm{Pd}]$, does not allow us to go beyond this type of mean-field approach. With these caveats in mind,
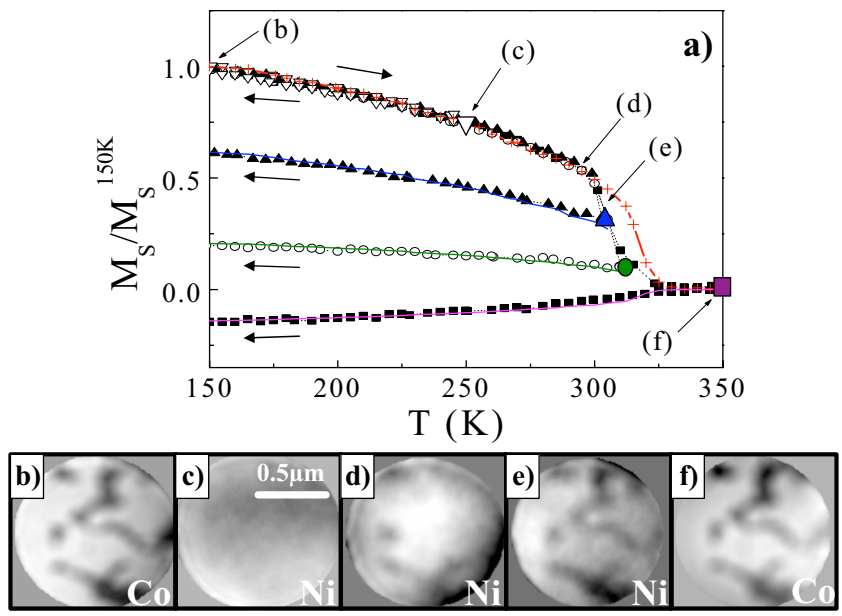

FIG. 4. (Color online) (a) Remanent magnetization during temperature cycles from $150 \mathrm{~K}$ to $T_{\max }$ after trapping a domain state in the hard layer at $150 \mathrm{~K}$, where $T_{\max }=250$ (open triangles), 300 (full triangles), 310 (circles), and $350 \mathrm{~K}$ (squares). The crosses represent the remanent magnetization with no domains in the $[\mathrm{Co} / \mathrm{Pd}]$ hard layer. The solid lines correspond to simulations as described in the text. (b),(f) 1.2- $\mu \mathrm{m}$-diameter FTH image of the magnetic domain configuration at the Co $L_{3}$ edge for remanence at 150 and $350 \mathrm{~K}$, respectively. (c)-(e) Remanent magnetic domain configuration at the Ni $L_{3}$ edge at 250, 290, and $300 \mathrm{~K}$.

leading to uncertainties of the simulated nucleation fields (estimated to $\pm 100 \mathrm{Oe}$ ), the calculated values qualitatively reproduce the trend of a reduction in nucleation field due to the stripe domains as well as the changes in the hysteresis loop shapes [Fig. 3(b)]. Nevertheless, thermal variations of $\Delta H_{N}$ cannot be quantitatively reproduced by considering only soft layer parameter changes [Fig. 3(a)]. We believe that thermal effects in the soft layer do not only lead to an effective reduction of the magnetization, exchange, and anisotropy as a function of temperature (Fig. 1), but also initiate kinetics of magnetization hopping over local energy barriers.

Finally, we take advantage of the temperature dependence in the competition between the intrinsic soft layer parameters and the dipolar interaction by demonstrating domain duplication during temperature cycling. First, at a sample temperature of $150 \mathrm{~K}$, we reverse about $50 \%$ of the hard layer magnetization, introducing the domain pattern shown in Fig. $4(\mathrm{~b})$, and subsequently measure the $[\mathrm{CoNi} / \mathrm{Pd}] / \mathrm{Pd} /[\mathrm{Co} / \mathrm{Pd}]$ magnetization at remanence as a function of temperature [Fig. 4(a)]. After saturating the soft layer at $3 \mathrm{kOe}$, the external field is released to remanence again. Subsequently starting at $150 \mathrm{~K}$, the temperature is cycled at remanence between $150 \mathrm{~K}$ and four temperatures $T_{\max }(250,300,310$, and $350 \mathrm{~K}$ ). After increasing the temperature beyond $290 \mathrm{~K}$, we observe an irreversible reduction in magnetization when cooling back down to $150 \mathrm{~K}$. Using FTH imaging during the temperature cycles, we first verify that the hard multilayer domain state remains unaltered within the temperature range of 150-350 K [Figs. 4(b) and 4(f)]. Then we image the soft layer domain state at the $\mathrm{Ni} L_{3}$ edge at 250, 290, and $300 \mathrm{~K}$ [Figs. 4(c)-4(e)]. While at $250 \mathrm{~K}$ the soft layer remains uniformly saturated, at 290 and $300 \mathrm{~K}$, domains are progressively replicated [Fig. 4(d) and 4(e)]. The FTH field of view 
is only microscopic. To characterize the domain replication rate as a function of $T_{\max }$ on the macroscopic scale, we calculate, from the VSM measurements, the percentage of nonreversed domains that fits the descending branches of the temperature cycle [Fig. 4(a)]. We use the soft layer remanent magnetization curve measured for a uniform hard layer state as a reference (100\% of up domains). We reproduce the magnetization variations as a function of temperature after $T_{\max }=300,310$, and $350 \mathrm{~K}$, considering $80 \%, 61 \%$, and $47 \%$ of [CoNi/Pd] up domains respectively [Fig. 4(a)]. The good agreement between the fits and the experimental data confirms the formation of a stable soft layer domain state via dipolar duplication when the system is cooled back down to $150 \mathrm{~K}$.

In conclusion, we present a direct visualization of stray field induced domain duplication in an all-perpendicular anisotropy system consisting of a hard/Pd/soft multilayer system, where the two magnetic layers are exchange decoupled via a thick Pd interlayer. While varying the external magnetic field, as well as the sample temperature, we use layer resolv- ing soft x-ray imaging techniques and directly show the influence of the hard layer magnetic domain configuration on the soft layer magnetization reversal. The competition between the intrinsic soft layer properties and the dipolar interaction is revealed during temperature dependent studies by tuning the soft layer Curie point well below the hard layer Curie point. Micromagnetic simulations reproduce qualitatively the temperature dependent changes of the soft layer hysteresis due to dipolar interactions. Finally, we demonstrate experimentally the concept of thermally driven domain duplication at remanence.

We thank M-Y. Im and K. Chen for help with experiments, and B. D. Terris and W. F. Schlotter for helpful discussions. T. Hauet was partially supported by a general Lavoisier grant. The soft x-ray microscope was supported by the Director, Office of Science, Office of Basic Energy Sciences, Materials Sciences and Engineering Division, of the U.S. Department of Energy.
${ }^{1}$ S. Gider, B.-U. Runge, A. C. Marley, and S. S. P. Parkin, Science 281, 797 (1998).

${ }^{2}$ M. Albrecht, G. Hu, A. Moser, O. Hellwig, and B. D. Terris, J. Appl. Phys. 97, 103910 (2005).

${ }^{3}$ B. Rodmacq, V. Baltz, and B. Dieny, Phys. Rev. B 73, 092405 (2006).

${ }^{4}$ S. Wiebel, J.-P. Jamet, N. Vernier, A. Mougin, J. Ferre, V. Baltz, B. Rodmacq, and B. Dieny, Appl. Phys. Lett. 86, 142502 (2005).

${ }^{5}$ J. Hohlfeld, Th. Gerrits, M. Bilderbeek, Th. Rasing, H. Awano, and N. Ohta, Phys. Rev. B 65, 012413 (2001), and references therein.

${ }^{6}$ H. Awano, S. Imai, M. Sekine, M. Tani, N. Ohta, K. Mitani, N. Takagi, H. Noguchi, and M. Kume, IEEE Trans. Magn. 36, 2261 (2000).

${ }^{7}$ P. Fischer, D. H. Kim, W. Chao, J. A. Liddle, E. H. Anderson, and D. T. Attwood, Mater. Today 9, 26 (2006).

${ }^{8}$ S. Eisebitt, J. Lüning, W. F. Schlotter, M. Lörgen, O. Hellwig, W.
Eberhardt, and J. Stöhr, Nature (London) 432, 885 (2004).

${ }^{9}$ S. Eisebitt, M. Lörgen, W. Eberhardt, J. Lüning, and J. Stöhr, Appl. Phys. A: Mater. Sci. Process. 80, 921 (2005).

${ }^{10}$ Q. Meng, W. P. van Drent, J. C. Lodder, and Th. J. A. Popma, J. Magn. Magn. Mater. 156, 296 (1996).

${ }^{11}$ P. Fischer, Curr. Opin. Solid State Mater. Sci. 7, 173 (2003).

${ }^{12}$ W. Chao, B. H. Harteneck, J. A. Liddle, E. H. Anderson, and D. T. Attwood, Nature (London) 435, 1210 (2005).

${ }^{13}$ M. S. Pierce, C. R. Buechler, L. B. Sorensen, J. J. Turner, S. D. Kevan, E. A. Jagla, J. M. Deutsch, T. Mai, O. Narayan, J. E. Davies, K. Liu, J. Hunter Dunn, K. M. Chesnel, J. B. Kortright, O. Hellwig, and E. E. Fullerton, Phys. Rev. Lett. 94, 017202 (2005).

${ }^{14}$ J. Grabis, A. Nefedov, and H. Zabel, Rev. Sci. Instrum. 74, 4048 (2003).

${ }^{15}$ G. Bertotti, Hysteresis in Magnetism (Academic Press, San Diego, 1998), p. 178. 